\title{
A Random Walk Approach for Light Scattering in Material
}

\author{
Klaus Simon and Beat Trachsler
}

Swiss Federal Laboratories for Materials Testing and Research, EMPA, St. Gall, Switzerland

Klaus.Simon@empa.ch

\begin{abstract}
Understanding reflection is one of the key competences in graphic arts industry. A very popular approach was given by KUBELKA and MUNK [1] who derived a simple relationship between the scattering and absorption coefficients and the overall reflectance. This paper presents an alternative approach which describes the behavior of light in matter as a special kind of random walk.
\end{abstract}

Keywords: Random Walk, Kubelka-Munk, Light Scattering, First-Passage Time Probability, Narayana Numbers, Catalan Numbers, Chebyshev Polynomials

\section{Introduction}

Seeing means perceiving light from an external source which was scattered in a material medium and reemitted toward the observer's eye. Thereby colors are the result of wavelength dependent absorption processes. The physical basis of color as molecular or atomic phenomenon is state-of-the-art in science, both in experimental and in theoretical respect. But to the authors knowledge, the deriving of macroscopic applications therefrom, for instance printing, is not really successful because of the huge complexity of corresponding models. Accordingly, state-of-the-art technologies like color management systems represent entirely empirical approaches which can be considered as scientific capitulation to the practical problems of color prediction. Therefore the ongoing challenge of modeling scattering affects is to optimize the compromise between simplicity of the use and the accuracy of prediction.

For printing the widely used theory of KUBELKA and MUNK dates back to 1931 [1]. In the course of time this original theory was extended by several authors, see PHILIPS-INVERNIZZI [2] for a recent survey. In SIMON [3] the authors started the trial to give a stochastic interpretation of KUBELKA and MUNK's theory. Now, this paper presents the next step on this trail containing several extensions and, in particular, a combinatorial analysis of the underlying distributions and solutions for the refectance and the transmittance in case of finite layer thickness.

The original work of KUBELKA and MUNK proposed a system of differential equations induced by a simplified model of light propagation, similar to the concepts of SCHUSTER [4] and GUREVIČ [5]. A horizontal colorant layer with thickness $D$ is considered. We suppose that the paint material is homogeneous. Then any light inside the layer traveling in any direction can be divided into its vertical and horizontal components. For simplification, the horizontal components are ignored. Therefore only two 
vertical fluxes of light have to be considered: a downward flux $i$ and an upward flux $j$. Let $i x_{x}$ and $j_{x}$ be the intensities of these two fluxes at the distance $x, x \leq D$, from the bottom surface. Then the theory of KUBELKA and MUNK is based on the assumption that the fractional amount of light lost by absorption (scattering) between $x$ and $x+\mathrm{d} x$ is given by $K \mathrm{~d} x(S \mathrm{~d} x)$, where $K(S)$ is denoted by absorption (scattering) coefficient. Light absorbed between $x$ and $x+\mathrm{d} x$ is lost, but light scattered from direction $i$ is added to $j$ and vice-versa ${ }^{1}$. On these assumptions we obtain

$$
\frac{\mathrm{d} i_{x}}{\mathrm{~d} x}=(K+S) \cdot i_{x}-S \cdot j_{x} \quad \text { and } \quad \frac{\mathrm{d} j_{x}}{\mathrm{~d} x}=S \cdot i_{x}-(K+S) \cdot j_{x}
$$

which implies

$$
\frac{\mathrm{d} r_{x}}{\mathrm{~d} x}=S \cdot r_{x}^{2}-2 \cdot(K+S) \cdot r_{x}+S
$$

where $r_{x}=j_{x} / i_{x}$ is called the reflectance ratio. Now, the aim is the determination of the reflectance $R=r_{0}$. A first solution of equation (2) arises if the layer is so thick that further increases in thickness do not significantly change its reftectance, i.e. $\mathrm{d} r_{x} / \mathrm{d} x=0$. In this situation let $R_{\infty}$ be the value of $\left(r_{0}\right)_{D \rightarrow \infty}$ then

$$
R_{\infty}=1+\frac{K}{S}-\sqrt{\left(1+\frac{K}{S}\right)^{2}-1}
$$

or equivalent

$$
\frac{K}{S}=\frac{\left(1-R_{\infty}\right)^{2}}{2 R_{\infty}}
$$

which is the original result of KUBELKA and MUNK [1]. Well-known variants are proposed by FoOTE [6], [7]

$$
R_{\infty}=1+\frac{K}{S}-\sqrt{\frac{K^{2}}{S^{2}}+\frac{2 K}{S}}=c-\sqrt{c^{2}-1}=c-c^{\prime} \quad \text { with } c=1+K / S \text { and } c^{\prime}=\sqrt{c^{2}-1}
$$

and by SAUNDERSON [8]. Later, KUBELKA [9] solved equation (2) on the additional assumption that the reffectance of the background (bottom) is known. Let $R_{g}$ be this fraction. Then the general solution is ${ }^{2}$

$$
R_{D}=\frac{1-R_{g} \cdot\left(c-c^{\prime} \cdot \operatorname{coth}\left(c^{\prime} S D\right)\right)}{c-R_{g}+c^{\prime} \cdot \operatorname{coth}\left(c^{\prime} S D\right)} .
$$

The difference $R_{\infty}-R_{D}$ is due to the transmission of the incident energy flux through the layer of thickness $D$ where the transmittance $T$ is defined as ratio of transmitted to incident light. For a disappearing scattering coefficient the transmittance can asymptoticly be written as $T \approx e^{-K D}$ well-known as Beer's law. In the classical concept of KUBELKA and MUNK Fresnel-effects of the interface between outside and inside of the colorant layer are ignored. In order to keep the mathematical constraints as simple as possible we proceed in the same way. On the other hand, Fresnel-effects can be taken into account by SAUNDERSON-corrections [8, pp.728], see also [10], [11] for recent treatments of this topic.

So far so good. Due to its simplicity, the theory of KUBELKA and MUNK is in common usage for industrial applications. However, this concept has also disadvantages, in particular, from a theoretical

\footnotetext{
1 note that $K$ and $S$ are functions of wavelengths
}

2 coth means the hyberbolic cotangent 
point of view. Please note that the central parameter, the reffectance, is defined as the ratio of reffected to incident radiation energy flux ${ }^{3}$, usually expressed as a percentage. In other words, the reflectance is a probability, namely the probability that a photon penetrating the surface will be reemitted subsequently. From this point of view, KUBELKA and MUNK describe implicitly a probability process treating expected values as fluxes and applying some kind of Chebyshev's inequality to estimate the reffectance. The authors interest is to make this process explicit. In order to guarantee that inadequacies of the model are due to the assumptions set down in it integration as part of the model evaluation has to be avoided. Therefore only a discrete probability model should be applied. This has the additional advantages that the combinatorial background of the model can be worked out exactly, functional relationships to other applications can be verified easier and the defining parameters have a clear macroscopic interpretation.

The rest of the paper is organized as follows. First, we introduce a new Markov chain modeling a KUBELKA-MUNK-like scattering process and study its combinatorial properties. Next, this Markov chain is simplified to a special random process similar to a random walk. Then we show that the reffectance can be determined as an adapted first-passage time problem. In particular, the case of finite thickness $D$ is solved in terms of Chebyshev polynomials of the second kind. We complete with some remarks.

\section{Photon Motion as a Markov Chain}

The classic case of a discrete-time birth-and-death process ${ }^{4}$ can be described as a sequence of random variables $X_{t}, t \in \mathbb{N}$, assuming the states $\ell=0,1,2, \ldots$ with probability $P_{t, \ell}$. We suppose that the process starts at state 0 and epoch 0 , hence $P_{0,0}=1$. Direct transitions to state $\ell$ are only possible from state $\ell-1$ and $\ell+1$. The probability that such a transition takes place between epoch $t-1$ and $t$ is noted by 5

$$
\lambda_{t-1, l-1}=\operatorname{Pr}\left(X_{t}=\ell \mid X_{t-1}=\ell-1\right) \quad \text { and } \quad \beta_{t-1, \ell+1}=\operatorname{Pr}\left(X_{t}=\ell \mid X_{t-1}=\ell+1\right) .
$$

Consequently, the process satisfies

$$
P_{t, \ell}=\left(1-\lambda_{t-1, \ell}-\beta_{t-1, \ell}\right) \cdot P_{t-1, \ell}+\lambda_{t-1, \ell-1} \cdot P_{t-1, \ell-1}+\beta_{t-1, \ell+1} \cdot P_{t-1, \ell+1} \cdot
$$

A random walk w $^{6}$ is a discrete time birth-and-dead process with $\lambda_{t, \ell}=\lambda, \beta_{t, \ell}=\beta$ and $\lambda+\beta=1$, which simplifies (7) to $P_{t, \ell}=\lambda \cdot P_{t-1, \ell-1}+\beta \cdot P_{t-1, \ell+1}$. A random walk with absorbing barriers ${ }^{7}$ at $B_{1} \leq 0$ and $B_{2} \geq 0$ is a random walk which stops at epoch $t$ if $B_{1} \leq X_{t^{\prime}} \leq B_{2}$ for $0 \leq t^{\prime}<t$ and $X_{t} \in\left\{B_{1}, B_{2}\right\}$.

At first we have to model the traveling of a photon as a random walk with absorbing barriers. Thereby we suppose that the photon moves in every discrete time step from the discrete level $\ell$ to $\ell+1$ or $\ell-1$. The entrance level is $\ell=0$. The absorbing barrier $B_{1}=-1$ represents the outside of the colorant layer. The second barrier $B_{2}=d+1$ stands for the bottom of the layer and, consequently, $d \geq 1$ for its thickness. The nontrivial part of our concept is the transition probability, which has to express the scattering of light. Usually, the transition probability is a function of the state $\ell$ and/or the time $t$. In our case, this is wrong because a traveling photon has a direction "up" or "down" independent of $\ell$ or $t$. From this point of view, scattering has to be understood as arbitrarily changing the direction from "up" to "down" or vice-versa.

\footnotetext{
3 for a given wavelength

4 see FELLER[12]

${ }^{5} \operatorname{Pr}(A \mid B)$ means the probability of event $A$ assuming the event $B$

6 This concept can easily be extended to higher dimensions.

7 the ruin problem

8 and only these directions, in analogy to KUBELKA and MUNK
} 
Therefore, we need a new explicit state in the Markov chain to describe that the transition probability depends on the photon's direction. Our choice is the number $h$ of turnabouts from the beginning of the motion at $t=0$ where even values of $h$ stand for the photon's direction "up" and odd values represent "down". Let $P_{t, \ell, h}$ be the probability that the photon is at epoch $t$ after $h$ turnabouts in state $\ell$. Then equation (7) mutates formally to

$$
\begin{aligned}
& P_{t, \ell, h}=\quad \lambda_{t-1, \ell-1, h} P_{t-1, \ell-1, h}+\mu_{t-1, \ell-1, h-1} P_{t-1, \ell-1, h-1} \\
&+\alpha_{t-1, \ell+1, h} P_{t-1, \ell+1, h}+\beta_{t-1, \ell+1, h-1} P_{t-1, \ell+1, h-1}
\end{aligned}
$$

with initial condition

$$
P_{0,0,0}=1
$$

Thereby the transition probabilities ${ }^{9}$

$$
\lambda_{t, \ell, h} \stackrel{\text { def }}{=}\left\{\begin{array} { l l } 
{ p } & { \text { if } h \text { even } } \\
{ 0 } & { \text { if } h \text { odd } , }
\end{array} \quad \mu _ { t , \ell , h } \stackrel { \text { def } } { = } \left\{\begin{array}{ll}
0 & \text { if } h \text { even } \\
q & \text { if } h \text { odd }
\end{array}\right.\right.
$$

and

$$
\alpha_{t, \ell, h} \stackrel{\text { def }}{=}\left\{\begin{array} { l l } 
{ 0 } & { \text { if } h \text { even } } \\
{ p } & { \text { if } h \text { odd } , }
\end{array} \quad \beta _ { t , \ell , h } \stackrel { \text { def } } { = } \left\{\begin{array}{ll}
q & \text { if } h \text { even } \\
0 & \text { if } h \text { odd }
\end{array}\right.\right.
$$

are determined by the assumption that the photon continues in its direction between $t-1$ and $t$ with probability $p$ or a turnabout because of scattering effects takes place with probability $q=1-p$. Of course, the constant $q$ goes with $S$. Hence, we call $q$ the scattering probability.

Next we consider the absorption, where we suppose that the layer material absorbs the photon during one time unit with probability $A$, the so-called absorption probability. In the case of absorption the process is determined immediately.

Additionally, we assume that scattering and absorption of a photon are independent events. From a physical point of view this assumption is not absolutely correct. But on the other hand, our assumption is not unusual, in particular, for small scattering and absorption coefficients ${ }^{10}$, and it is more reasonable than KUBELKA-MUNK's one which allows also in the case of total absorption a positive reftectance.

However, the real motivation for assuming independence between scattering and absorption is an easier treatment of the reffectance $R$, the main object of our interest. Obviously, $R$ is equivalent to the probability that the photon leaves again the colorant layer before it was absorbed in the material. But more interestingly, this probability can be expressed as a classical problem connected to random walks, namely the first-passage time problem. Let $w_{t}(A)$ be the probability that the photon leaves the layer at time $t$ or in random walk terminology the first visit to -1 takes place at the $t$-th step, see FELLER [12] for a detailed description. Then the refectance $R$ is given by

$$
R=w_{0}(A)+w_{1}(A)+w_{2}(A)+\cdots \quad .
$$

Now, by induction on $t$ we infer from the independence of the absorption events $w_{t}(A)=w_{t}(0) \cdot(1-A)^{t}$ which together with (12) leads to

$$
R=\sum_{t=0}^{\infty} w_{t}(A)=\sum_{t=0}^{\infty} w_{t}(0) \cdot(1-A)^{t}=R(1-A)
$$

\footnotetext{
9 Please note that, related to our transition probabilities, in comparison with the traditional defi nition the role of $\alpha$ and $\beta$ are permanently exchanged.

10 the practical relevant cases
} 
where $R(z)$ is the generating function for the first-passage time probability ignoring absorption. In other words, the reffectance $R$ is implicitly determined by the generating function of the corresponding firstpassage time problem and, accordingly, an explicit treatment of absorption effects can be omitted.

\section{The Distribution}

In this section we consider only layers of infinite thickness $(d=\infty)$. Thereby, our aim is the probability $w_{t}=w_{t}(0)$ that the first visit to -1 takes place at the $t$-th step. Since a traveling photon starting at level 0 needs an even number of steps for coming back to 0 , the level -1 is only reachable for odd $t$, hence $w_{t}=0$ for $t$ even. For $w_{1}$ the first and last step of the photon is from 0 to -1 which is by the initial condition (9) a turnabout and therefore we obtain $w_{1}=q$. On the other hand, for all other odd $t, t \geq 3$, the last step to -1 is not a change in direction and has probability $p$. Then summing up over all possible values of $h$ leads to

$$
w_{t}=p \cdot P_{t-1,0,1}+p \cdot P_{t-1,0,3}+\cdots+p \cdot P_{t-1,0, t-2} \text {. }
$$

The calculation of the first values of $w_{t}$ by equation (8) shows

$$
\begin{aligned}
w_{3} & =1 p^{2} q \\
w_{5} & =1 p^{2} q^{3}+1 p^{4} q \\
w_{7} & =1 p^{2} q^{5}+3 p^{4} q^{3}+1 p^{6} q \\
w_{9} & =1 p^{2} q^{7}+6 p^{4} q^{5}+6 p^{6} q^{3}+1 p^{8} q \\
w_{11} & =1 p^{2} q^{9}+10 p^{4} q^{7}+20 p^{6} q^{5}+10 p^{8} q^{3}+1 p^{10} q \\
& \cdots
\end{aligned}
$$

A glance at the encyclopedia of integer Sequences discloses that the coefficients in this development are known as Narayana numbers ${ }^{11}$, see [13], which can be formally inferred from the distribution

$$
P_{t, \ell, h}= \begin{cases}p^{t} & \text { if } h=0 \text { and } t=\ell \\
\frac{2(t+h \ell)}{t^{2}-\ell^{2}}\left(\begin{array}{c}
\frac{t-\ell}{2} \\
\frac{h+1}{2}
\end{array}\right)\left(\begin{array}{c}
\frac{t+\ell}{2} \\
\frac{h-1}{2}
\end{array}\right) p^{t-h} q^{h} & \text { if } h \text { odd and } t+\ell \text { even } \\
\frac{2 h \ell}{t^{2}-\ell^{2}}\left(\begin{array}{c}
\frac{t-\ell}{2} \\
\frac{h}{2}
\end{array}\right)\left(\begin{array}{c}
\frac{t+\ell}{2} \\
\frac{h}{2}
\end{array}\right) p^{t-h} q^{h} & \text { if } h \geq 2 \text { even and } t+\ell \text { even } \\
0 & \text { otherwise. }\end{cases}
$$

Induction on $t$ using equation (8) leads in case of an even $h$ to

$$
\begin{aligned}
P_{t, \ell, h} & \stackrel{(8)}{=} p \cdot P_{t-1, \ell-1, h}+q \cdot P_{t-1, \ell-1, h-1} \\
& =\frac{2 h(\ell-1)}{(t-1)^{2}-(\ell-1)^{2}}\left(\begin{array}{c}
\frac{t-\ell}{2} \\
\frac{h}{2}
\end{array}\right)\left(\begin{array}{c}
\frac{t+\ell}{2}-1 \\
\frac{h}{2}
\end{array}\right) p^{t-h} q^{h}+\frac{2(t-1+(h-1)(\ell-1))}{(t-1)^{2}-(\ell-1)^{2}}\left(\begin{array}{c}
\frac{t-\ell}{2} \\
\frac{h}{2}
\end{array}\right)\left(\begin{array}{c}
\frac{t+\ell}{2}-1 \\
\frac{h}{2}-1
\end{array}\right) p^{t-h} q^{h} \\
& \left.=[\frac{2 h(\ell-1)}{(t-1)^{2}-(\ell-1)^{2}} \underbrace{\left(\begin{array}{c}
\frac{t+\ell}{2}-1 \\
\frac{h}{2}
\end{array}\right)}_{=\frac{t+\ell-h}{t+\ell}\left(\begin{array}{c}
\frac{t+\ell}{2} \\
\frac{h}{2}
\end{array}\right)}+\frac{2(t-1+(h-1)(\ell-1))}{(t-1)^{2}-(\ell-1)^{2}} \underbrace{\frac{h}{2}-1}_{=\frac{t+\ell}{t+\ell}\left(\begin{array}{c}
\frac{t+\ell}{2} \\
\frac{h}{2}
\end{array}\right)})\right]
\end{aligned}
$$

11 usually defi ned as $\frac{1}{n}\left(\begin{array}{c}n \\ i\end{array}\right)\left(\begin{array}{c}n \\ i-1\end{array}\right)$ 


$$
=\frac{2 h \ell(t+\ell-2)}{\left(t^{2}-\ell^{2}\right)(t+\ell-2)}\left(\begin{array}{c}
\frac{t-\ell}{2} \\
\frac{h}{2}
\end{array}\right)\left(\begin{array}{c}
\frac{t+\ell}{2} \\
\frac{h}{2}
\end{array}\right) p^{t-h} q^{h} .
$$

The odd case follows in a similar way:

$$
\begin{aligned}
& P_{t, \ell, h} \stackrel{(8)}{=} \quad p \cdot P_{t-1, \ell+1, h}+q \cdot P_{t-1, \ell+1, h-1} \\
& =\frac{2(t-1+h(\ell+1))}{(t-1)^{2}-(\ell+1)^{2}}\left(\begin{array}{c}
\frac{t-\ell}{2}-1 \\
\frac{h+1}{2}
\end{array}\right)\left(\begin{array}{c}
\frac{t+\ell}{2} \\
\frac{h-1}{2}
\end{array}\right) p^{t-h} q^{h}+\frac{2(h-1)(\ell+1)}{(t-1)^{2}-(\ell+1)^{2}}\left(\begin{array}{c}
\frac{t-\ell}{2}-1 \\
\frac{h-1}{2}
\end{array}\right)\left(\begin{array}{c}
\frac{t+\ell}{2} \\
\frac{h-1}{2}
\end{array}\right) p^{t-h} q^{h} \\
& =[\frac{2(t-1+h(\ell+1))}{(t-1)^{2}-(\ell+1)^{2}} \underbrace{\left(\begin{array}{c}
\frac{t-\ell}{2}-1 \\
\frac{h+1}{2}
\end{array}\right)}+\frac{2(h-1)(\ell+1)}{(t-1)^{2}-(\ell+1)^{2}} \underbrace{\left(\begin{array}{c}
\frac{t-\ell}{2}-1 \\
\frac{h-1}{2}
\end{array}\right)}]\left(\begin{array}{c}
\frac{t+\ell}{2} \\
\frac{h-1}{2}
\end{array}\right) p^{t-h} q^{h} \\
& =\frac{t-\ell-h-1}{t-\ell}\left(\begin{array}{c}
\frac{t-\ell}{2} \\
\frac{h+1}{2}
\end{array}\right) \quad=\frac{h+1}{t-\ell}\left(\begin{array}{c}
\frac{t-\ell}{2} \\
\frac{h+1}{2}
\end{array}\right) \\
& =\frac{2(t+h \ell)(t-\ell-2)}{\left(t^{2}-\ell^{2}\right)(t-\ell-2)}\left(\begin{array}{c}
\frac{t-\ell}{2} \\
\frac{h+1}{2}
\end{array}\right)\left(\begin{array}{c}
\frac{t+\ell}{2} \\
\frac{h-1}{2}
\end{array}\right) p^{t-h} q^{h} .
\end{aligned}
$$

Especially for $t$ even, $\ell=0$ and $h$ odd we observe from (15)

$$
P_{t, 0, h}=\frac{2}{t}\left(\begin{array}{c}
\frac{t}{2} \\
\frac{h+1}{2}
\end{array}\right)\left(\begin{array}{c}
\frac{t}{2} \\
\frac{h-1}{2}
\end{array}\right) p^{t-h} q^{h}
$$

implying together with (13) and (14)

$$
R=q \cdot(1-A)+\sum_{\substack{t=3 \\
t \text { odd }}}^{\infty}\left(\sum_{\substack{h=1 \\
h \text { odd }}}^{t-2} \frac{2}{t-1}\left(\begin{array}{c}
\frac{t-1}{2} \\
\frac{h+1}{2}
\end{array}\right)\left(\begin{array}{c}
\frac{t-1}{2} \\
\frac{h-1}{2}
\end{array}\right) p^{t-h} q^{h}\right) \cdot(1-A)^{t}
$$

This explicit expression for the refectance allows now combinatorial interpretations in connection with lattice path combinatorics [13] or catwalks [14]. Note that summing up the coefficients of $P_{t, \ell, h}$ over all values of $h$ leads to the table 22.5 of [15] induced by $U_{n}(x)$, the Chebyshev polynomials of the second kind. However for an intuitive comparison with the result of KUBELKA and MUNK (3) an additional closed formula of $R$ is desirable.

\section{An Adapted Random Walk}

In the foregoing section we have shown that our scattering problem can successfully be treated as a Markov chain. But this Markov chain does not harmonize well with the classical solution of a first-passage time problem, namely a direct calculation of the generating function by the involved convolution equation. For that reason we offer now an equivalent random process, no longer a Markov chain, which can be adapted to the traditional argumentation. This new process is also suitable in the case of finite thickness $d$.

The new concept is based on an alternative formulation of the transition probability without accounting the turnabouts. Let $X_{t}$ be again the random variable related to the position of the photon $\ell=0,1,2, \ldots$ In contradiction to the classical case

$$
\lambda_{t-1, l-1}=\operatorname{Pr}\left(X_{t}=\ell \mid X_{t-1}=\ell-1\right)
$$


we define the transition probability now as

$$
\lambda_{t-1, \ell-1} \stackrel{\text { def }}{=} \begin{cases}p & \text { if } X_{t-1}=\ell-1 \wedge X_{t-2}=\ell-2 \\ q & \text { if } X_{t-1}=\ell-1 \wedge X_{t-2}=\ell\end{cases}
$$

and

$$
\beta_{t-1, \ell+1} \stackrel{\text { def }}{=} \begin{cases}p & \text { if } X_{t-1}=\ell+1 \wedge X_{t-2}=\ell+2 \\ q & \text { if } X_{t-1}=\ell+1 \wedge X_{t-2}=\ell\end{cases}
$$

respectively. Note that a change in direction is now indicated by the events

$$
X_{t-2}=\ell, X_{t-1}=\ell-1, X_{t}=\ell \quad \text { and } \quad X_{t-2}=\ell, X_{t-1}=\ell+1, X_{t}=\ell .
$$

Because of our non-Markovian transition probability the initial condition $P_{0,0}=1$ has to be extended to

$$
P_{0,0}=1 \quad \text { and } \quad P_{-1,-1}=1
$$

or

$$
P_{0,0}=1 \quad \text { and } \quad P_{-1,1}=1 .
$$

Both versions are possible and have their own right. The condition (18) describes the physical situation when the photon is getting into the layer. The second will be induced by recurrent events. Correspondingly,

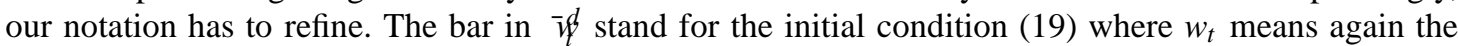
"first visit to -1 at $t$ "-probability and $d$ indicates the layer thickness which can be $\infty$. Other variables are characterized in the same way.

Again we get $w_{t}^{d}=0=w_{\text {f }}$ for $t$ even and immediately from the definition we obtain

$$
w_{1}^{d}=q \quad \text { and } \quad \text { 惮 }=p
$$

which induces

$$
\bar{w}_{p}^{d}=\frac{p}{q} \cdot w_{1}^{d} .
$$

Note that for $t \geq 3$ the probabilities $i_{p}$ and $w_{t}^{d}$ are connected by an opposite identity

$$
\underline{w}=\frac{q}{p} \cdot w_{t}^{d}
$$

which can be verified easily by induction on $t$. Let us now consider the case of infinite layer thickness. For odd $t \geq 3$ we observe

$$
w_{t}^{\infty}=p \cdot w_{1}^{\infty} \cdot \ddot{w}_{-2}^{\infty}+p \cdot w_{3}^{\infty} \cdot \ddot{w}_{-4}^{\infty}+\cdots+p \cdot w_{t-2}^{\infty} \cdot \dddot{w}_{1}^{\infty}
$$

To show (23) the event leading to $w_{t}^{\infty}$ is divided into a set of mutually exclusive events $A_{2}, A_{4}, A_{6}, \ldots$. Clearly, for $t \geq 3$ the photon goes to 1 in the first step. Therefore, there exists a smallest subscript $i$, $1<i<t$, with $X_{i}=0$. For every $i$ the event $A_{i}$ contains three blocks of trials.

block $B_{1}$. The photon goes to 1 in the first step. 
block $B_{2}$. The photon needs exactly $i-1$ further trials to reestablish the initial situation.

block $B_{3}$. It takes exactly $t-i$ further trials to reach -1 .

These three events depend on non-overlapping blocks of transitions with, by definition, fixed values of $X_{0}$, $X_{1}, X_{i-1}$ and $X_{i}$. Every block forms a recurrent probability of type $w_{t}^{\infty}$ or $\not \dddot{p}$. From the definition we get $\operatorname{Pr}\left(B_{1}\right)=p$. The events in $B_{2}$ assuming $X_{0}=0 \wedge X_{1}=1$ have the probabilities $w_{i-1}^{\infty}$ and the events in $B_{3}$ assuming $X_{i-1}=1 \wedge X_{i}=0$ can be expressed as $\ddot{\not}_{-i}$. So the probability of the simultaneous realization of all three events is given by the product

$$
\operatorname{Pr}\left(B_{1} \wedge B_{2} \wedge B_{3}\right)=\underbrace{\operatorname{Pr}\left(B_{1}\right)}_{=p} \times \underbrace{=\underbrace{\operatorname{Pr}\left(B_{2} \mid B_{1}\right)}_{=\operatorname{Pr}\left(B_{2} \mid X_{0}=0 \wedge X_{1}=1\right)} \times \underbrace{\operatorname{Pr}\left(B_{3} \mid X_{i-1}=1 \wedge X_{i}=0\right)}_{=\dddot{M}_{-i}}}_{=w_{i-1}^{\infty}} .
$$

Summing up over all possible $i$ completes (23). The recurrence (23) can now be simplified by

$$
\stackrel{-\infty}{\stackrel{(20)}{=}} p \quad \text { and } \quad \stackrel{\infty}{\not} \stackrel{(22)}{=} \frac{q}{p} \cdot w_{t}^{\infty} \quad \text { for } t \geq 3
$$

implying

$$
w_{t}^{\infty}=q \cdot w_{1}^{\infty} \cdot w_{t-2}^{\infty}+\cdots+q \cdot w_{t-4}^{\infty} \cdot w_{3}^{\infty}+p^{2} \cdot w_{t-2}^{\infty}
$$

or equivalent

$$
w_{t}^{\infty}=\left(p^{2}-q^{2}\right) \cdot w_{t-2}^{\infty}+q \cdot\left(w_{1}^{\infty} \cdot w_{t-2}^{\infty}+\cdots+w_{t-2}^{\infty} \cdot w_{1}^{\infty}\right)
$$

Remark. From (25) on the one hand and (14), (15) on the other hand it is now easy to verify $w_{t}=w_{t}^{\infty}$ and therefore the equivalence of our two probability models.

Now we are able to determine the generating function $R(z)$. We set $w_{0}^{\infty}=0$ for convenience and $w_{1}^{\infty}=q$ was given in (20). Next note that

$$
w_{1}^{\infty} \cdot w_{t-2}^{\infty}+\cdots+w_{t-2}^{\infty} \cdot w_{1}^{\infty}
$$

is the $(t-1)$-th coefficient of $\left(R^{\infty}(z)\right)^{2}$. Hence, we infer from (25)

$$
\begin{aligned}
R^{\infty}(z)-q \cdot z=\sum_{t=2}^{\infty} w_{t}^{\infty} \cdot z^{t} & =\sum_{t=2}^{\infty}\left[\left(p^{2}-q^{2}\right) \cdot w_{t-2}^{\infty}+q \cdot\left(w_{1}^{\infty} \cdot w_{t-2}^{\infty}+\cdots+w_{t-2}^{\infty} \cdot w_{1}^{\infty}\right)\right] \cdot z^{t} \\
& =\left(p^{2}-q^{2}\right) \cdot z^{2} \cdot R^{\infty}(z)+q \cdot z \cdot\left(R^{\infty}(z)\right)^{2} .
\end{aligned}
$$

The physically reasonable solution of this quadratic equation is

$$
R^{\infty}(z)=\frac{1+\left(q^{2}-p^{2}\right) \cdot z^{2}-\sqrt{\left(1+\left(q^{2}-p^{2}\right) \cdot z^{2}\right)^{2}-4 \cdot q^{2} \cdot z^{2}}}{2 \cdot q \cdot z} .
$$

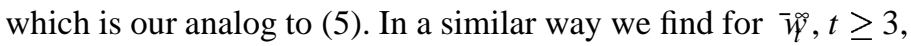

$$
\bar{w}^{\infty}=q \cdot w_{1}^{\infty} \cdot \stackrel{-}{w}_{-2}+q \cdot w_{3}^{\infty} \cdot \stackrel{-}{\psi}_{-4}+\cdots+q \cdot w_{t-2}^{\infty} \cdot \stackrel{-}{w}^{\infty}
$$

and as a consequence

$$
\bar{R}^{\infty}(z)-p \cdot z=q \cdot z \cdot R^{\infty} \cdot \bar{R}^{\infty}(z)
$$


or equivalent

$$
\bar{R}^{\infty}(z)=\frac{p \cdot z}{1-q \cdot z \cdot R^{\infty}(z)}=\frac{1-\left(q^{2}-p^{2}\right) \cdot z^{2}-\sqrt{\left(1-\left(q^{2}-p^{2}\right) \cdot z^{2}\right)^{2}-4 \cdot p^{2} \cdot z^{2}}}{2 \cdot p \cdot z} .
$$

Note also that $\bar{R}^{\infty}(z)$ is obtained from $R^{\infty}(z)$ by exchanging $p$ and $q$. Further, the equations (26) together with (17) result in

$$
\frac{1+\left(q^{2}-p^{2}\right) z^{2}-\sqrt{\left(1+\left(q^{2}-p^{2}\right) z^{2}\right)^{2}-4 q^{2} z^{2}}}{2 q z}=q z+\sum_{i=1}^{\infty} \frac{z^{2 i+1}}{i} \sum_{j=0}^{i-1}\left(\begin{array}{c}
i \\
j+1
\end{array}\right)\left(\begin{array}{c}
i \\
j
\end{array}\right) p^{2(i-j)} q^{2 j+1} .
$$

Let us now turn to $\bar{w}$ for a bounded $d$. Trivially, $\bar{\nu}_{p}=p$ and $\bar{R}^{0}(z)=p z$. Then, by induction on $t$ we see

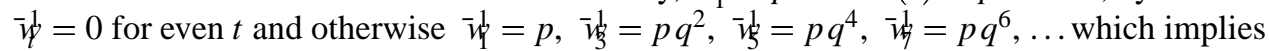

$$
\bar{R}^{1}(z)=p z+p q^{2} z^{3}+p q^{4} z^{5}+p q^{6} z^{7}+\cdots=\frac{p z}{1-q^{2} z^{2}}
$$

For $d \geq 2$ the middle part of the recurrent events (block $B_{2}$ ) in (27) have to be adapted to $d-1$, hence

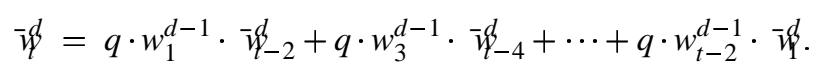

Then the consideration of $w_{1}^{d-1} \stackrel{(20)}{=} q$ and $w_{t}^{d-1} \stackrel{(22)}{=} \frac{p}{q} \cdot \eta^{d-1}$ shows

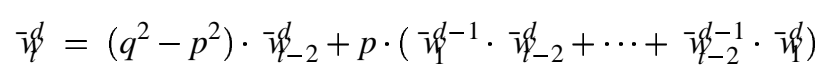

which together with

$$
u=\frac{1-\left(q^{2}-p^{2}\right) z^{2}}{p z}
$$

leads to

$$
\bar{R}^{d}(z)=\frac{p \cdot z}{1-\left(q^{2}-p^{2}\right) \cdot z^{2}-p \cdot z \cdot \bar{R}^{d-1}(z)}=\frac{1}{u-\bar{R}^{d-1}(z)} .
$$

The special type of continued fractions given in (32) have solutions as elementary variants of Chebyshev polynomials, see [16, p. 156], which can be verified as follows. Let $U_{n}(x)$ be the Chebyshev polynomials of the second kind given by $U_{0}(x)=1, U_{1}(x)=2 x$ and

$$
U_{n}(x)=2 x \cdot U_{n-1}(x)-U_{n-2}(x) \text { for } n \geq 2 .
$$

Then the polynomials $K_{n}(u, a, b)$ defined by

$$
K_{0}(u, a, b)=a, \quad K_{1}(u, a, b)=b
$$

and

$$
K_{n}(u, a, b)=u \cdot K_{n-1}(u, a, b)-K_{n-2}(u, a, b)
$$

satisfy

$$
K_{n}(u, a, b)=a \cdot U_{n}\left(\frac{u}{2}\right)+(b-a u) \cdot U_{n-1}\left(\frac{u}{2}\right)
$$


for $n \geq 1$ which is easy to see by induction on $n$. Now the standard solution of (32), see [17], is now given by

$$
\bar{R}^{d}(z)=\frac{K_{d}(u, a, b)}{K_{d+1}(u, a, b)}
$$

with

$$
u=\frac{1-\left(q^{2}-p^{2}\right) z^{2}}{p z}, \quad a=1, \quad b=\frac{1}{p z} .
$$

On the other hand, in the same way as (27) the recurrence (30) implies

$$
\bar{R}^{d}(z)=\frac{p \cdot z}{1-q \cdot z \cdot R^{d-1}(z)}
$$

which together with (32) and (37) induces

$$
R^{d}(z)=\frac{\left(q^{2}-p^{2}\right) \cdot z}{q}+\frac{p}{q} \cdot \bar{R}^{d}(z)=\frac{\left(q^{2}-p^{2}\right) \cdot z}{q}+\frac{p \cdot K_{d}(u, a, b)}{q \cdot K_{d+1}(u, a, b)} .
$$

Finally we come to the transmittance $T$. Let $v_{t}^{d}$ be the probability that the photon's first visit to $d+1$ takes place at the $t$-th step and let $T^{d}(z)$ be the corresponding generating function. Then in analogy to the refectance $R$ the transmittance is implicitly determined by $T=T^{d}(1-A)$. Thereby the foregoing concepts allow also the calculation of $T^{d}(z)$. Obviously we observe $v_{1}^{0}=p, v_{t}^{1}=p^{2} q^{t-2}$ for even $t \geq 2$ and $v_{t}^{1}=0$ for odd $t$. Again, the case $d \geq 2$ can be reduced to $d-1$ implying

$$
\begin{aligned}
& v_{d+1}^{d}=p^{d+1}, \\
& v_{t}^{d}=v_{d}^{d-1} \cdot \bar{\psi}_{-d}+v_{d+2}^{d-1} \cdot \bar{\psi}_{-(d+2)}+\cdots+v_{t-1}^{d-1} \cdot \bar{\nu}_{\varphi}
\end{aligned}
$$

and $v_{t}^{d}=0$ for $t<d$ or $t=d, d+2, d+4, \ldots$. From this we infer

$$
T^{0}(z)=p z=\bar{R}^{0}(z), \quad T^{1}(z)=\frac{p^{2} z^{2}}{1-q^{2} z^{2}}=\bar{R}^{0}(z) \cdot \bar{R}^{1}(z)
$$

and for $d \geq 2$

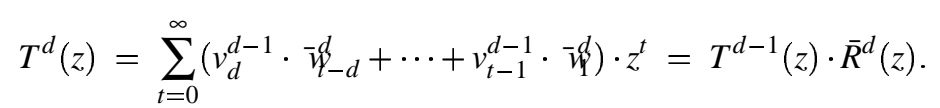

Iteration of (42) together with (37) leads to

$$
T^{d}(z)=\frac{K_{d}(u, a, b)}{K_{d+1}(u, a, b)} \cdot \frac{K_{d-1}(u, a, b)}{K_{d}(u, a, b)} \cdots \frac{K_{1}(u, a, b)}{K_{2}(u, a, b)} \cdot \frac{K_{0}(u, a, b)}{K_{1}(u, a, b)}=\frac{1}{K_{d+1}(u, a, b)} .
$$

\section{Final Remark}

Because of the unusual transition probability a random walk approach for light scattering in material was not obvious. But it seems that an adaption of the classical language of random walks to our application is natural and fruitful. In connection with this, thanks to the referees for their helpful hints. Although the discrete solution avoids some of the contradiction of the classical KUBELKA-MUNK-theory and improves its accuracy the new concept appears more as an evolution than as a revolution. On the other hand, some interesting questions are offered by our analysis, for instance, the general role of Chebyshev polynomials in connection with scattering and transmission. 


\section{References}

[1] P. Kubelka and F. Munk. Ein Beitrag zur Optik der Farbanstriche. Zeits. f. techn. Physik, 12:593-601, 1931.

[2] B. Philips-Invernizzi, D. Dupont, and C. Cazé. Bibliographical review for reftectance of diffusing media. Optical Engineering, 40(6):1082-1092, June 2001.

[3] K. Simon. A Stochastik Interpretation of Kubelka-Munk. In CGIV 2002: First European Conference on Colour in Graphics, Image and Vision, pages 468-472, University of Poitiers, France, April $2-5,2002$. IS\&T.

[4] A. Schuster. Radiation through a foggy atmosphere. Astrophys. Journal, 21(1):1-22, 1905.

[5] M. Gurevič. Über eine rationelle Klassifikation der lichtstreuenden Medien - flache Schicht von lichtsreuenden Teilchen im Vakuum. Phys. Z., 31:753-763, 1930.

[6] W. Foote. An investigation of the optical scattering and absorption coefficients of dyed handsheets and the application of the I.C.I. system of color specification to these handsheets. Paper Trade J., 108(TS125), March 91939.

[7] W. Foote. An investigation of the optical scattering and absorption coefficients of dyed handsheets. Paper Trade J., 109(TS31), Dec. 211939.

[8] J. L. Saunderson. Calculation of the Color of Pigmented Plastics. J. Opt. Soc. Amer., 32(4):727-736, 1942.

[9] P. Kubelka. New Contributions to the Optics of Intensely Light-scattering Materials, Part I. J. Opt. Soc. Amer., 38:448-457, 1948.

[10] S. Mourad, P. Emmel, K. Simon, and R. Hersch. Extending Kubelka-Munk's Theory with Lateral Light Scattering. In D. Weiss and M. Yuasa, editors, NIP 17: International Conference on Digital Printing Technologies, pages 469-473, Fort Lauderdale, Florida, September 30 - October 5, 2001. IS\&T.

[11] F. Berg. Isotrope Lichtstreuung in Papier - Neue Überlegungen zur Kubelka-Munk-Theorie. PhD thesis, Technische Hochschule Darmstadt, April 1997.

[12] W. Feller. An Introduction to Probability Theory and Its Applications. John Wiley \& Sons, 1968.

[13] T. V. Narayana. Lattice Path Combinatorics with Statistical Applications. University of Toronto Press, 1979.

[14] R. Guy. Catwalks, Sandsteps and Pascal Pyramids. Journal of Integer Sequences, 3(Article 00.1.6), 2000.

[15] M. Abramowitz and I. Stegun. Handbook of Mathematical Functions. United States Government Printing Office, 1964.

[16] P. Flajolet. Combinatorial aspects of continued fractions. Discrete Mathematics, 32:125-161, 1980.

[17] O. Perron. Die Lehre von den Kettenbrüchen. Teubner, 1954. 
